Patrick T. Mather

Dale S. Pearson $\dagger$

Ronald G. Larson

Dong-Feng $\mathrm{Gu}$

Alexander M. Jamieson

\section{The origin of stress-oscillation damping during start-up and reversal of torsional shearing of nematics}

Received: 11 February 1997

Accepted: 10 June 1997

Dr. P.T. Mather ( )

Wright Laboratory/Materials Directorate

2941 P St., Ste 1

Wright Patterson AFB,

Ohio 45433-7750, USA

E-mail: Matherpt@ml.wpafb.af.mil

R. G. Larson

Department of Chemical Engineering

University of Michigan

Ann Arbor, Michigan, 48109, USA

D.-F. Gu

Rockwell Science Center

Thousand Oaks, California 91360, USA

A. Jamieson

Department of Macromolecular Science

Case Western Reserve University

Cleveland, Ohio 44106, USA

$\leftarrow$ Deceased
Abstract Using a controlled-temperature shear cell mounted on a polarizing microscope, we observe the behavior of nematic $4,4^{\prime}$-n $n$-octyl-cyanobiphenyl $(8 \mathrm{CB})$ during start-up and reversal of shearing in a torsional parallel-plate geometry and correlate this behavior with rheological measurements. During the startup, a sequence of birefringent rings, or "twist walls", are observed that originate at the sample edge and propagate radially inward. Each twist wall is a thin region in which the director is twisted out of the plane of the velocity and velocitygradient directions. The radial variation of in-plane orientation can be explained by the variation of strain in the parallel-plate device. A high-
Ericksen-number solution of the Leslie-Ericksen equations predicts a damped oscillatory shear stress response which agrees quantitatively with the measured stress oscillations out to an edge strain of around 50 . The damping of the stress oscillations is due to the nonuniformity of strain in the parallel-plate geometry. On reversal of the flow, if the strain, $\gamma$, is smaller than about 500 units, the damping of stress oscillations is reversed; this correlates with an outward radial migration of twist walls. When $\gamma>500$, disclinations nucleate and spoil the reversibility of stress damping.

Key words Liquid crystal tumbling $-8 \mathrm{CB}$

\section{Introduction}

Recently, we investigated the shear-flow behavior of a small-molecule liquid crystal with $\alpha_{3}>0\left(4,4^{\prime}-n\right.$-octylcyanobiphenyl or $8 \mathrm{CB}$ ) in mechanical and optical rotational rheometers ( $\mathrm{Gu}$ et al., 1993; Gu and Jamieson, 1994; Mather et al., 1996). In the mechanical measurements, the time-dependent shear stress after start-up of a shearing flow with initially homeotropic director orientation showed an oscillatory behavior, in agreement with predictions of Ericksen's transversely isotropic fluid (TIF) model. A representative result is reproduced in Fig. 1. The observed damping of shear stress (or apparent viscosity) with strain is distinctly different from the damping associated with elastic torques, as calculated by Burghardt and Fuller using the Leslie-Ericksen theory (Burghardt and
Fuller, 1990), in that there is no accompanying gradual rise in the shear stress, or apparent viscosity, with increased strain. Prior to the current work, explanations for the damping of the shear stress oscillations included rotation of the director away from the shear plane (Rey, 1993), the plane containing the velocity and velocity-gradient directions, and/or the creation of randomly located disclinations. However, no direct observations supporting these explanations were made. Optical observations on transient shear flow of $8 \mathrm{CB}$ reported here indicate another explanation, described shortly.

Previous work on torsional shear flow of $8 \mathrm{CB}$ (Skarp et al., 1981; Carlsson and Skarp, 1986) has revealed that, under certain conditions, a pattern of concentric walls is generated from the successive creation of "twist walls" at the sample edge which move radi- 


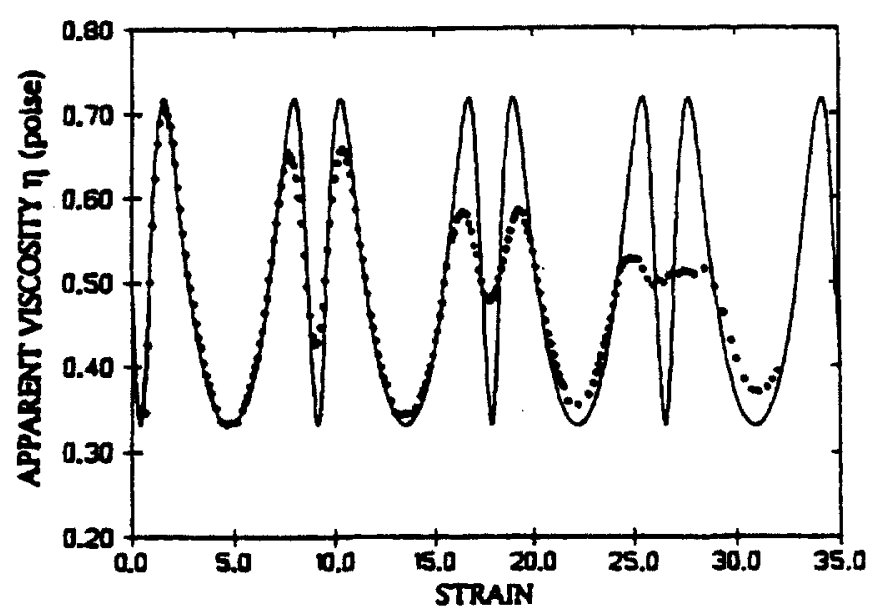

Fig. 1 Apparent viscosity measured during shear start-up of homeotropically aligned $8 \mathrm{CB}$ in a cone and plate rheometer (symbols) along with a fit of the data using Ericksen's transversely isotropic fluid model (from Gu and Jamieson, 1994)

ally inward until a steady state is achieved. The authors interpreted these walls as defect surfaces where an inplane tumbling instability ${ }^{1)}$, takes place such that the wall defect, or "twist wall", separates regions with different director profiles. After cessation of flow and subsequent application of an a.c. electric field normal to the plates (the dielectric anisotropy for $8 \mathrm{CB}$ is positive) the pattern transforms into bright and dark rings of similar width when viewed between crossed polarizers. The authors interpreted the dark rings as regions between the twist walls which had relaxed to the homeotropic anchoring condition with the aid of the applied field. The bright rings were interpreted as twist walls which had relaxed and broadened, under the applied field, taking on director orientations out of the plane of shear. The authors noted that such a pattern occurs if a high rotation speed is turned on suddenly from rest. The relevant dimensionless shear rate by which "high" and "low" shear rates can be distinguished is the Ericksen number, $\mathrm{Er}=\dot{\gamma} h^{2} \gamma_{1} / K_{3}$, where $\dot{\gamma}$ is the shearing rate, $h$ is the sample thickness, $\gamma_{1}$ is the rotational viscosity, and $K_{3}$ is the Frank bend elastic constant.

Steady-state calculations (Manneville, 1981; Carlsson, 1984) in which the director is constrained to the shear plane predict that as Er increases the director profile undergoes "tumbling transitions" where the director profile "jumps". These jumps occur in such a way that, throughout the gap, the director rotates through a finite angle (which depends on the vertical position) with an infinitesimal increase in Er in the case of a gradually increasing Er. These calculations indicate that the director

\footnotetext{
1) By "in-plane tumbling instability" we refer to the prediction of the Leslie-Ericksen equations, for the case where the director is constrained to the shear plane, so that the steady-state director profiles, $\theta(y)$, undergo sudden jumps when $\mathrm{Er}$ is increased past certain values.
}

orientation at the sample mid-plane rotates through an angle close to $\pi$, when a tumbling transition occurs.

In this paper, we use in-situ shearing-flow observations to explore the nature of twist walls formed during start-up and reversal of homeotropically-aligned $8 \mathrm{CB}$ and to investigate the correlation between twist-wall formation and rheological properties.

\section{Experimental}

Materials

4,4'-n-octylcyanobiphenyl was purchased from E.M. Industries and used as received. Sample purity was confirmed using clearing point measurements and the clearing point was found to be within $0.1^{\circ} \mathrm{C}$ of the accepted value $40.1^{\circ} \mathrm{C}$. The experiments were performed at $T=37.0^{\circ} \mathrm{C}$, where the material is a tumbling nematic; i.e. the Leslie viscosity, $a_{3}$, is larger than zero.

\section{Rheological and optical observations}

Mechanical rheology was performed using a Rheometrics RFS 8500 fluids rheometer using parallel steel disks $50 \mathrm{~mm}$ in diameter which had been treated with lecithin for homeotropic alignment of the nematic director. Previous experience with this alignment method indicated that uniform alignment of the director could be achieved (for $8 \mathrm{CB}$ at the test temperature of $37^{\circ} \mathrm{C}$ ) in $30 \mathrm{~min}$ and these conditions were used for the current experiments. The sample thickness used was $200 \mu \mathrm{m}$ to duplicate the conditions of the optical experiments. The lower theometer disk was rotated at various speeds yielding various edge shear rates. The direction of shear was reversed after the accumulated strain reached various values.

Optical observations were performed using a custom rheological microscope described in detail elsewhere (Mather, 1994; Mather et al., 1996b). In this apparatus, a fluid sample was sheared between two glass disks, $38 \mathrm{~mm}$ in diameter, which were thermostated using foil heaters. The bottom disk was rotated relative to the fixed top disk whose displacement from the lower plate was controlled using a three-micrometer kinematic mount. As with the mechanical experiments, the nematic director was made initially homeotropic using surface treatment with lecithin. Optical observations were made using a CCD camera with short focal-length lens focused on the fluid sample through an optically transparent radial slit of the rheometer which extended from the cell center to the sample meniscus. The slit had a width of $3.8 \mathrm{~mm}$. With this set-up, the optical field of view covered a length of approximately $3 / 4$ the 
disk radius. The sample was viewed between crossed polarizers with the incident light being polarized in the flow direction.

Polarizing light microscopy observations were performed using a long working distance $20 \times$ objective lens with an analyzer crossed with the polarizer, the polarizer being oriented in the flow direction. Conoscopic observations were used to examine the director configuration near the twist walls. For these observations, a laser beam was expanded to approximately $1.5 \mathrm{~cm}$, then polarized $45^{\circ}$ from the flow direction and focused (condensed) onto the sheared sample with a total included angle of approximately $25^{\circ}$. The diverging cone of light above the sample was passed through an analyzer crossed with the polarizer $\left(135^{\circ}\right.$ orientation) and projected on an opaque screen located $5 \mathrm{~cm}$ above the sample film so that positions within the conoscopic patterns corresponded to unique light trajectories through the sample. This use of convergent monochromatic illumination yields a spatial resolution in the velocityvorticity plane of approximately $75 \mu \mathrm{m}$. Each conoscopic pattern, therefore, represents a pattern reflecting orientation over an area $75 \mu \mathrm{m}$ in diameter. The fringe patterns observed in the conoscopic patterns, each corresponding to an order of birefringence, were then interpreted to yield the average director orientation across the sample thickness (Bloss, 1961; Mather et al., 1996a).

\section{Results and discussion}

As discussed in a previous paper (Mather et al., $1996 \mathrm{c}$ ), when the angular velocity of the bottom disk of the parallel-plates rotational rheometer is started from rest at a "large" value $\left(\mathrm{Er}_{R}>1000\right.$, where $\mathrm{Er}_{R}$ is the Ericksen number at the sample edge), and when the sample is viewed between crossed polarizers with the incident light polarized in the flow direction, a progression of highly birefringent rings, oriented in the flow direction, are observed to propagate inward from the edge. It was shown in that paper that under conditions of $\theta=0.05 \mathrm{rad} / \mathrm{s}$, and a sample thickness of $200 \mu \mathrm{m}$, the first of the sequence of rings propagates with an initial velocity of $0.86 \mathrm{~mm} / \mathrm{s}$, with the velocity decreasing with decreasing radial position.

Shown in Fig. 2 are observations of the propagation of the birefringent circular rings under the same conditions described above, but with optical viewing of the entire radial-view slit. Again, the crossed-polarizers are arranged with the incident light polarized in the flow direction. With this configuration, areas where light is extinguished by the analyzer represent regions where the director is either in the plane of shear or in the velocity gradient-vorticity plane. As seen in Fig. $2 \mathrm{a}$, the first
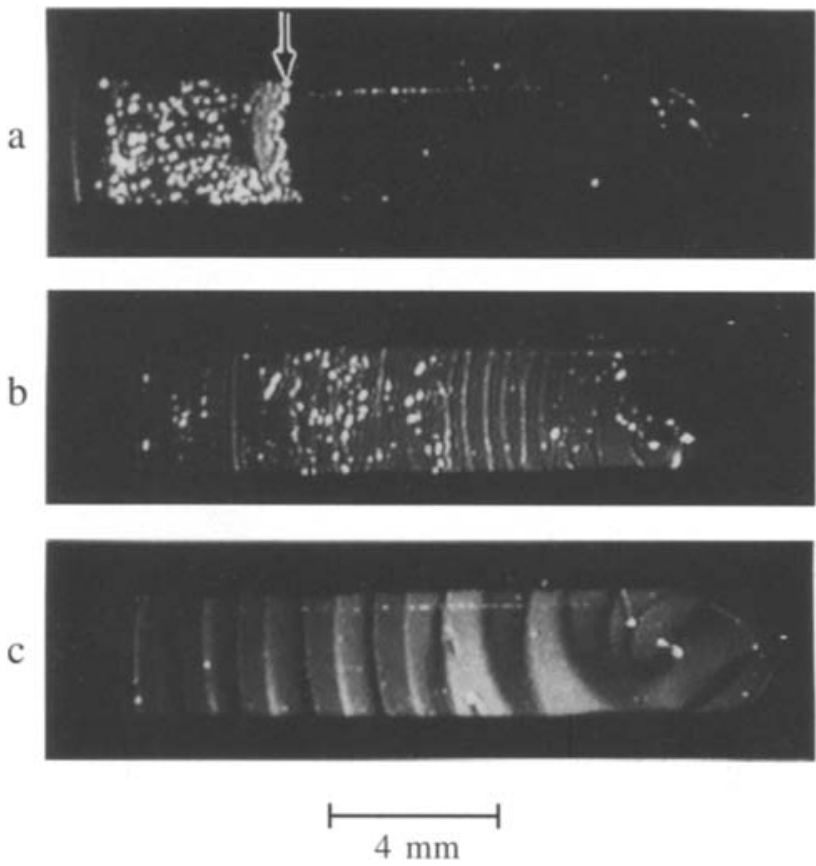

Fig. 2 Full optical slit view of shear start-up at $0.05 \mathrm{rad} / \mathrm{s}$ : (2a) the first birefringent ring (see arrow) propagating inward, $15 \mathrm{~s}$ after shear start-up, (2b) after 2 min of steady shearing, yielding an accumulated edge strain of 500, and (2c) several minutes after shear had stopped at the conditions of ( $2 \mathrm{~b}$ ). Sample thickness is $225 \mu \mathrm{m}$ and vertical slit width is $3.8 \mathrm{~mm}$

ring, indicated by the arrow, leaves many defects in its wake. These defects, appearing as white spots at this low magnification, quickly collapse and disappear. After prolonged shearing (approximately $2 \mathrm{~min}$ ), as seen in $2 \mathrm{~b}$, many birefringent rings (appearing thinner in Fig. $2 b$ than in 2 a) have propagated toward the center and a pseudo-steady-state is achieved, with the ring velocities becoming negligible. Consistent with the local microscopic observations of the ring propagation is the observation that, aside from locations very close to the rings and wake defects, the director remains in the plane of shear, as evidenced by the extinction of light. If the rheometer disk rotation is continued for a large number of edge strain units, on the order of 500 or larger, the regularity of the concentric birefringent rings is disrupted and the cell becomes filled with disclination lines, as is beginning to occur in Fig. $2 \mathrm{~b}$. However, if the rotation is stopped soon after the regular pattern of birefringent rings has been established, the texture transforms to concentric rings which are alternating dark/bright/dark/etc., when viewed between crossed polarizers, as shown in Fig. $2 \mathrm{c}$. This pattern shows the relaxation of the director pattern from the predominately in-plane configuration during shear to a pattern that contains significant out-of-plane director orientation, as 
Fig. 3 Time sequence of conoscopic images revealing the director orientation near the propagating birefringent ring. The sample is viewed from above with the lower plate moving downward, as indicated in the inset. Far ahead of the ring (a), the director is tipped and rotating in the shear flow direction within the shearing plane. As the ring propagation approaches the observation radius, the director twists out of the plane of shear (b) with a clockwise sense when viewed from above, and $\theta$ increases to nearly $\pi / 2$, while twisting away from the shear plane (c). Very close to the twist wall $(\mathrm{d}-\mathrm{f})$, the director has twisted to an orientation perpendicular to the shearing plane $(\phi=\pi / 2)$ with $\theta$ increasing from $\pi / 2$ to $\pi$. The disk rotation speed is $0.011 \mathrm{rad} / \mathrm{s}$, the sample thickness is $350 \mu \mathrm{m}$, and the viewing radius is $15 \mathrm{~mm}$. Times for each image, relative to $t=0$ at shear start-up, are: (a) 11 , (b) 20 , (c) 26 , (d) 29, (e) 31 , (f) $33 \mathrm{~s}$. Nail heads represent orientation into the page and the view is of the velocity (vertical)-vorticity (horizontal) plane
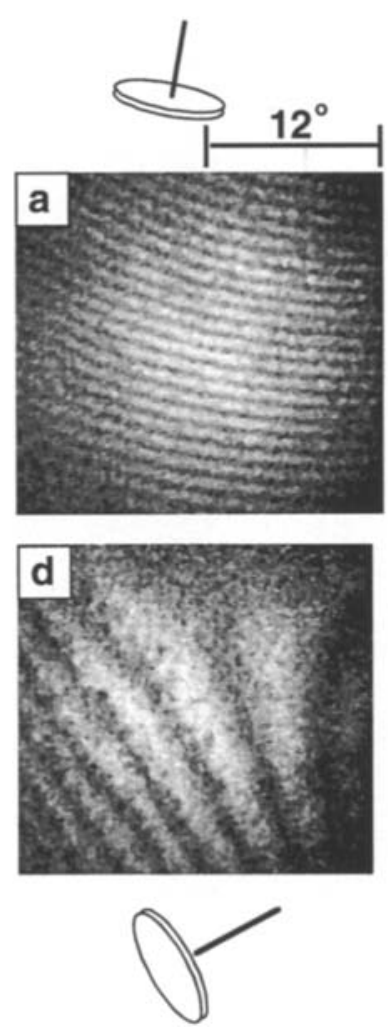
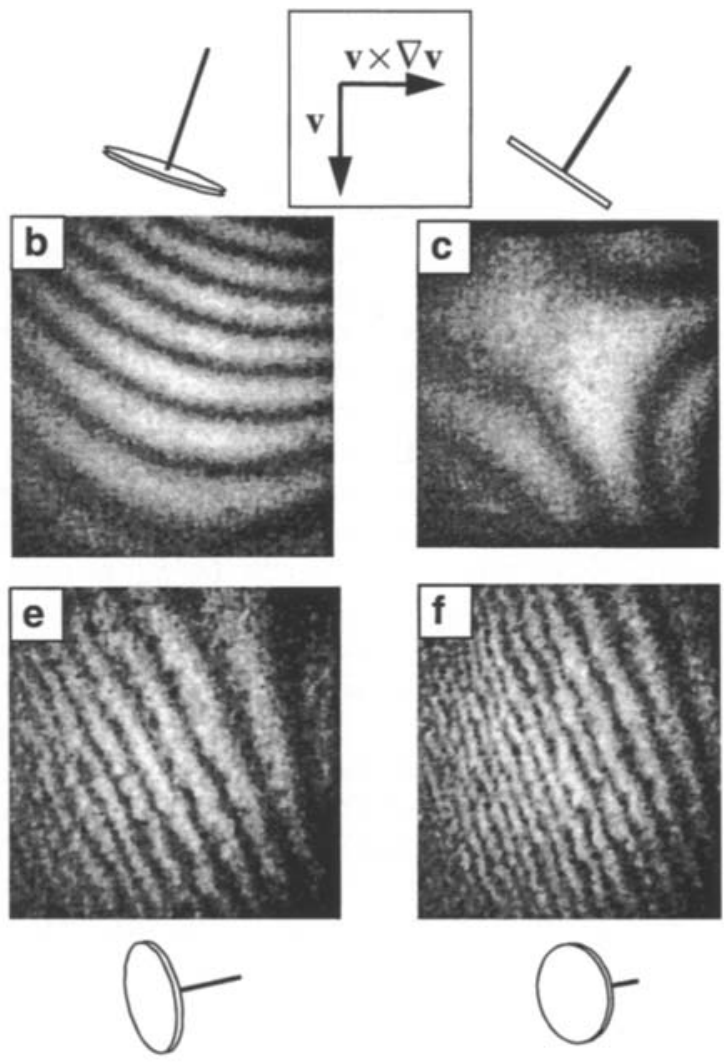

evidenced by light transmission through the crossed polarizers.

The configuration of the director near the birefringent rings was investigated using conoscopy with laser illumination and at a radial position $15 \mathrm{~mm}$ from the axis of rotation ( $4 \mathrm{~mm}$ from the sample edge). The conoscopic patterns are formed above the flow cell, with the bottom plate rotating counterclockwise, so that relative to the patterns the flow direction is downward and the velocity gradient direction is into the page. Because the rings propagate radially inward, the spatially varying director structure near them is most easily observed by fixing the position of conoscopic observation and observing the changes in director orientation as a ring passes. Since the rings appear to propagate radially without changing much, the director configuration at fixed position and different times presumably reflects the spatial dependence of the director configuration at fixed time. This interpretation is based on the observation that translation of the microscope at a velocity close to that of the ring with some fixed radial offset yields a conoscopic image that is relatively constant in time. Thus, the motion of the twist wall through the sample is that of a solitary wave.

To make meaningful conoscopic observations, it was necessary to choose a value for $\mathrm{Er}_{R}$ low enough so that the director was radially homogeneous over the conoscopic resolution of $75 \mu \mathrm{m}$, yet high enough so that the boundary layer ${ }^{2)}$ (Pikin, 1974) was a small fraction of the sample thickness. Only under these circumstances were we able to obtain well defined conoscopic interference fringes. This optimization of experimental conditions, for $h=350 / \mu \mathrm{m}$, yields $\mathrm{Er}_{R} \cong 354(\dot{\theta}=0.011 \mathrm{rad} / \mathrm{s})$. For this case, the boundary layer at each plate is calculated to be $45 \mu \mathrm{m}$ thick, such that the top and bottom boundary layers account for $25 \%$ of the sample thickness; i.e., the majority of the nematic fluid is uniformly oriented. Here, the boundary layer thickness is given by $\delta=h\left(2 /\left(\mathrm{Er}^{2} \cdot|\varepsilon|\right)\right)^{1 / 4}$, where $\varepsilon=a_{3} / a_{2}$ (Pikin, 1974).

For the following discussion, the angles $\theta$ and $\phi$ are used to describe the director orientation, averaged across the sample thickness, observed as a function of distance from the first twist wall. Similar results are observed between successive twist walls following the first. Here, $\theta$ measures the angle between velocity-gradient direction and the projection of the director onto the shearing plane, while $\phi$ measures the angle between the director and the shearing plane. Prior to shear flow,

\footnotetext{
2) Boundary layer refers to the region near shearing surfaces over which the director distorts from homeotropic boundary conditions to the hydrodynamically prescribed orientation.
} 
conoscopic images show that the sample was oriented homeotropically; i.e., a Maltese cross (a set of "isogyres") centered on the optic axis of the microscope was observed, along with a series of several concentric extinction rings ("isochromes") also centered on the optic axis.

Figure $3 \mathrm{a}$ shows that, far from the birefringent ring, before the ring has propagated inward to the viewing radius, the director is tipped mainly within the plane of shear $(\theta>0)$ with only a small out-of-plane tip $(\phi>0)$. Closer to the twist wall (Fig. $3 \mathrm{~b}$ ) $\theta$ increases toward a value of $\pi / 2$ (as indicated by the wide spacing and upward concavity of the interference fringes), and twists significantly out of the shear plane (Fig. 3c) yielding $\theta \sim \pi / 2, \phi \cong \pi / 4$. Very close to the twist wall (Fig. $3 \mathrm{~d}-$ $\mathrm{f})$, the director twists to an orientation which is nearly perpendicular to the shearing plane $(\phi=\pi / 2)$ and $\theta$ increases from $\pi / 2$ toward $\pi$; the director has thus rotated within the gradient-vorticity plane toward a homeotropic orientation at the center of the propagating twist wall. Along with each conoscopic image shown in Fig. 3 is a semi-quantitative "nail" representation of the director orientation observed. In this representation, the nail head represents director orientation into the page. Although the nematic director is a "head-less" vector, i.e. $-\boldsymbol{n}=\boldsymbol{n}$, we find this notation convenient for distinguishing between $\theta=\pi / 4$ and $\theta=3 \pi / 4$, for example. The transition of the conoscopic patterns between Fig. 3c and $\mathrm{d}$ occurs by both clockwise rotation and northeast translation of the pattern, indicating that both $\theta$ and $\phi$ are increasing with time. The transitions of the conoscopic patterns from Fig. $3 \mathrm{~d}$ to e and from $3 \mathrm{e}$ to $\mathrm{f}$ follow a similar evolution, but with only minor clockwise rotation and eastward translation of the interference fringes. Because we observe that the director twists substantially away from the shear plane near the birefringent ring, we henceforth refer to the rings as "twist walls".

The times at which each of conoscopic patterns were recorded with respect to the start of shearing are listed in the caption of Fig. 3. Additionally, we have examined the length scale over which the birefringent ring imparts an influence on the director orientation by computing the distance between propagating twist wall and the fixed observation position. These values were com puted based on twist wall propagation data, modeled to account for sample thickness, the angular velocity of the shearing disk, and the critical edge strains for twist wall formation, explained later in reference to Fig. 5 . The distances are (3a) 13.3, (3b) 8.53, (3c) 5.33, (3d) 3.73 , (3e) 2.67 , and $(3 \mathrm{f}) 1.6 \mathrm{~mm}$. Since the distance from the sample edge to the observation radius is $4 \mathrm{~mm}$, we see that Fig. $3 \mathrm{a}-\mathrm{c}$ represents observations made before the first twist wall has even formed at the edge of the shear cell. This means that for $\mathrm{Er}=354$, the presence of a twist wall is not required for significant twist of the director away from the shear plane. More- over, the length scale over which the twist wall may impart an influence, e.g. $3.73 \mathrm{~mm}$ for Fig. $3 \mathrm{~d}$, is surprisingly large. Therefore, it is possible that instead of twist walls forming at a critical strain with $\phi$ everywhere near zero and subsequently relaxing to minimize elastic energy, the twist walls may form at the sample edge only when the director has developed an orientation susceptible to twist wall formation.

The observation that the director leaves the shearing plane near the defect "core" by twisting toward a central homeotropic configuration has led us to refer to these defects as "twist-walls," following the terminology of Carlsson and Skarp (1986) and Skarp and coworkers (1981). However, the sense of twist we have observed is entirely different than that proposed by Carlsson et al., who suggested that the twist walls are positions where the director orientation within the shearing plane rotates due to the in-plane tumbling instability such that a sudden increase in director orientation is completely accommodated by director twist within the shearing plane. While the origin of the twist walls may be related to the in-plane tumbling instability on a coarse length scale, it appears that such a dramatic in-plane twist is not elastically favorable and a less dramatic director reorientation occurs instead. Indeed, we observed no evidence of the formation of a singular wall at all.

On the trailing (left) side of the twist wall, the director orientation appears to return to the shearing plane following the same reorientation observed in front of the ring but in reverse. We cannot say this with certainty, however, as it was exceedingly difficult to examine the difference between the phase of the in-plane director rotation before and after the described director reorientations. Nevertheless, orthoscopic observations are consistent with this conjecture, as we now describe. Shown in Fig. 4 is a micrograph "close-up" of the first of a series of radially propagating twist-walls (corresponding to the vertical streak in Fig. 2 a) as observed between crossed polarizers with the polarizer oriented in the flow direction, with similar images for subsequent twist walls. Under these viewing conditions, significant transmitted light corresponds to regions in the sample with out-of-plane director orientation, larger than 0 and less than $\pi / 2$. The two bright bands shown in Fig. 4, therefore, are regions within the twist-wall where the director is rotating through orientations with $0<\phi<\pi / 2$ (or $\pi / 2<\phi<\pi$ ). The dark band separating the two bright bands is a region within which the director is rotating through the homeotropic orientation, $\theta=n \pi$ $(n=0,1,2 \ldots)$, largely in the velocity-gradient-vorticity plane. This dark band corresponds roughly to the twistwall position examined with the conoscopic image in Fig. $3 \mathrm{f}$ and, together, they enable a distinction of a homeotropic orientation from a planar orientation with $\theta=\pi / 2$ and $\phi=\pi / 2$. 


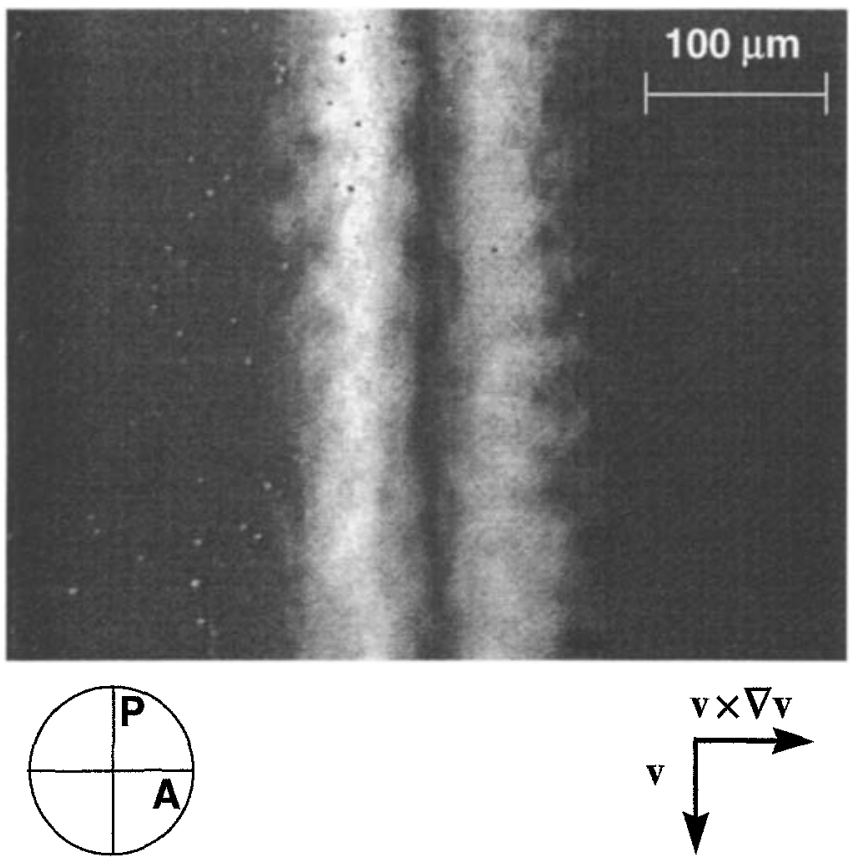

Fig. 4 Photomicrograph of a twist wall propagating radially inward (left-to-right). The sample is viewed with the polarizer and analyzer crossed and the polarizer oriented in the flow direction. The angular velocity is $0.03 \mathrm{rad} / \mathrm{s}$, the sample thickness is $200 \mathrm{~mm}$, and the sample is viewed near the edge $(r=17 \mathrm{~mm})$, yielding $\mathrm{Er} \cong 625$. The lower disk is rotating counter-clockwise and viewed at an azimuthal location such that the flow direction is downward, viewing the cell from above with the bottom plate rotating counter-clockwise

The width of brightness observed adjacent to the twist wall center in Fig. 4 is approximately $70 \mu \mathrm{m}$, in contrast to the length scale of close to $10 \mathrm{~mm}$ from the twist wall core shown in Fig. 3 over which the director was observed to rotate significantly out of the shearing plane. Two factors are involved in explaining this surprising difference. First, the value of Er at the observation radius changes from 354 in Fig. 3 to 626 in Fig. 4, and the observed change in length scale may be a result of decreasing elastic effects on increasing Er, especially considering that in the limit of low Er (Mather, 1996c) the director obtains a steady out-of-plane orientation with $\phi \cong \pi / 2$. Secondly, the length scale of director distortion as measured from intensity distribution using orthoscopic optics is subject to error in that the relationship between orientation angle and intensity is non-linear and the image contrast is sensitive to several experimental features. Conclusive evidence regarding the effect of Ericksen number on the length scale of director distortion near the propagating twist walls would only be possible by varying Er and making conoscopic observations as in Fig. 3. We hope to do this in the future.

In the absence of elastic effects, two-dimensional (2D) Leslie-Ericksen calculations of shear flow have predicted that in-plane tumbling events will occur at

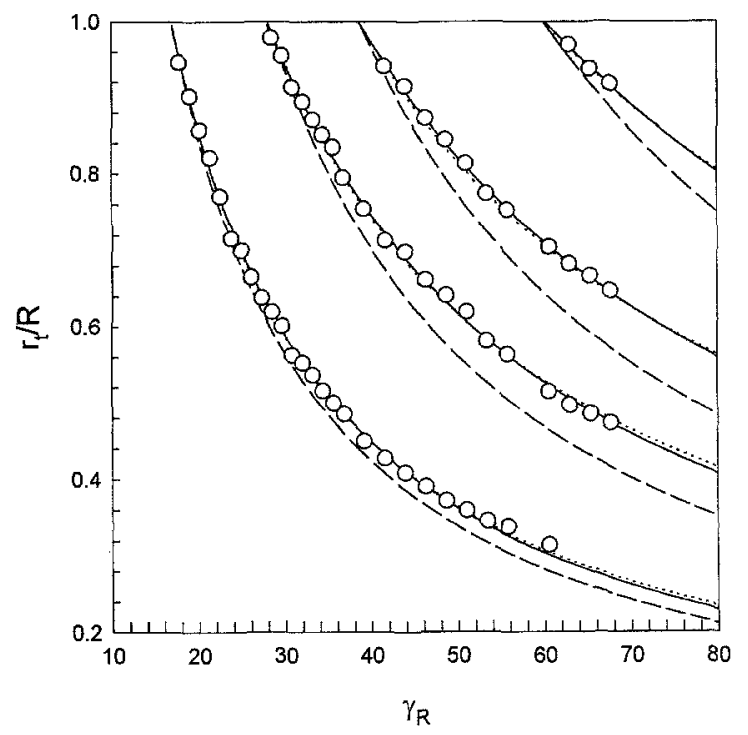

Fig. 5 Plot of dimensionless twist wall position, $\tilde{r}(t)$, versus edge strain, $\gamma_{R}$, for the first four twist walls created in torsional shear flow with $h=200 \mu \mathrm{m}$ and $\theta=0.75 \mathrm{rad} / \mathrm{s}$, yielding a large edge shear rate of $\dot{\gamma}=71 \mathrm{~s}^{-1}$, and an edge Ericksen number, $\mathrm{Ex}_{R}$ of 17500. Open circles are the data and the solid lines are the best fits using Eq. (2), dotted lines (close to solid lines) represent the best fits using Eq. (3), and dashed lines are best fits to the data using Eq. (2) with $a=1$ and the constraint of matching the solid line at $\tilde{r}(t)=1$ for each twist wall curve

critical values of strain. If the twist walls we observe originate from these events, and if the condition of minimal elasticity holds (i.e., Er $\gg 1$ ), we would except that the $i^{\text {th }}$ twist wall should propagate such that it passes through the radial position, $r^{i}(t)$, when the strain has reached the critical value,

$$
\gamma_{\text {crit }}^{i}=\frac{r^{i}(t) \dot{\theta} t}{h}
$$

yielding $r^{i}(t) \sim t^{-1}$. Here, $r^{i}(t)$ is the radial position of the $i^{\text {th }}$ twist wall in the cell, $\dot{\theta}$ is the angular velocity of the rotating disk, $t$ is time, and $h$ is the sample thickness. To test this, we video-taped twist-wall propagation at $\mathrm{Er}=17500$ for several twist walls produced during the first 150 edge strain units. The video tape images were then digitized using a Data Translations frame grabber (DT 3852) and analyzed for the radial position of the center of each of the bright rings as a function of time. The length scale of the image processing was calibrated using the known width of the optical slit visible in the images.

Shown in Fig. 5 are the results of the image processing plotted in dimensionless form as $\tilde{r}(t)$ versus $\gamma_{R}$, where $\tilde{r}(t) \equiv r(t) / R$ and $\gamma_{R}(t)$ is the edge strain. For this case, the sample thickness was $200 \mu \mathrm{m}$ and the disk rotation speed was $0.75 \mathrm{rad} / \mathrm{s}$, which yields an edge shear rate of $71 \mathrm{~s}^{-1}$, and a large edge Ericksen number $\mathrm{Er}_{R}=17500$. The first twist wall forms after 
The origin of stress-oscillation damping during start-up

Table 1 Parameters extracted from twist wall propagation data (Fig. 5)

\begin{tabular}{|c|c|c|c|c|}
\hline \multirow[t]{2}{*}{ Twist wall } & \multicolumn{2}{|c|}{$\tilde{r}(t)=\left(\frac{\gamma_{c}}{\gamma_{R}(t)}\right)^{a}$} & \multicolumn{2}{|c|}{$\tilde{r}(t)=\frac{\gamma_{c}^{\prime}}{\gamma_{R}(t)+\gamma_{R}^{0}}$} \\
\hline & $\gamma_{c}$ & $a$ & $\gamma_{c}^{\prime}$ & $\gamma_{R}^{0}$ \\
\hline 1 & 16.9 & 0.93 & 18.8 & 2.0 \\
\hline 2 & 28.1 & 0.84 & 35.8 & 8.0 \\
\hline 3 & 38.7 & 0.79 & 52.7 & 14.1 \\
\hline 4 & 60.0 & 0.75 & 80.6 & 20.4 \\
\hline
\end{tabular}

$0.25 \mathrm{~s}$, which is equivalent to an edge strain of 17.45 . In dimensionless form, Eq. (1) takes the form

$$
\tilde{r}(t)=\left(\frac{\gamma_{c}}{\gamma_{R}(t)}\right)^{\alpha},
$$

where $\gamma_{c}$ is a critical strain for twist wall formation and $a$ is an exponent near unity. The radial position of this first twist wall decreases with time in a manner well described by $\tilde{r}(t)=\gamma_{c} / \gamma_{R}(t)$. If the power law exponent, $\alpha$, is allowed to vary in the nonlinear regression of the data, however, an exponent $\alpha=0.933$ is measured. The second and subsequent twist walls propagate inward more slowly than would be expected of the form $\tilde{r}(t)=\gamma_{c} / \gamma_{R}(t)$. This is clearly seen in Fig. 5, in which the second twist wall, formed at $t=0.394 \mathrm{~s}$ or strain $=28.1$ units, follows a power law in edge strain with an exponent of 0.84 . This is shown as a solid line fit through the data. If the twist wall were to follow $\tilde{r}(t) \sim 1 / \gamma_{R}(t)$ behavior $(r(t) \sim 1 / t)$, the twist wall would propagate more quickly as is shown by the dashed curve forced to go through $\tilde{r}(t)=1$ at the critical strain determined from the fit with Eq. (2). An even larger deviation is shown for the third twist wall, while data for the fourth twist wall are too limited to extend the assessment, although the trend of increasing deviation appears to be continued.

An additional formula was found to fit the data as well as the power law does:

$$
\tilde{r}(t)=\frac{\gamma_{c}^{\prime}}{\gamma_{R}(t)+\gamma_{R}^{0}}
$$

and the results of fitting the data in this way are shown as dotted lines in Fig. 5, nearly superposing with the curves obtained by fitting with Eq. (2). Table 1 summarizes the parameters obtained from fitting the twistwall-propagation data with both Eq. (2) and (3). Although we are unable to ascribe precise physical meaning to the parameters $\gamma_{c}^{\prime}$ and $\gamma_{R}^{0}$, we observe that the quantity $\left(\gamma_{c}^{\prime}-\gamma_{R}^{0}\right)$ is numerically equivalent to $\gamma_{c}$, as is expected on equating Eqs. (2) and (3) at $\tilde{r}(t)=1$, and that the sequence of $\gamma_{c}^{\prime}$ values for twist walls one through four show the surprising progression of magnitude ratios of $1: 2: 3: 4$. For example, the value of $\gamma_{c}^{\prime}$ for the second twist wall is twice that of the first, the value of $\gamma_{c}^{\prime}$ for the third twist wall is three times that of the first, etc.

The deviation of the rate of twist-wall propagation from the $r(t) \sim 1 / t$ scaling $\left(r(t) \sim 1 / \gamma_{R}(t)\right.$ in dimensionless form) is most likely due to the influence of Frank elasticity. While Frank elasticity should initially be weak compared to viscous forces since the Ericksen number is large, the "winding up" of the director produced by director tumbling leads to an accumulation of elastic strain which might slow down the director rotation in the bulk, and hence slow down twist-wall propagation.

Prompted by the interesting result that shear stress oscillations during the start-up of shearing flow in cone-plate rheometry of $8 \mathrm{CB}$ return on flow reversal for not-too-high strains before reversal (Gu, Jamieson, and Wang, 1993), we now seek to examine whether or not twist-wall formation and propagation is reversible under analogous conditions in torsional plate-plate shear flow. To do this, we perform transient shear experiments in which a $200-\mu$ m-thick $8 \mathrm{CB}$ sample is aligned homeotropically and suddenly sheared with a wide range of rotation speeds yielding a wide range of $\mathrm{Er}_{R}$, the edge Ericksen number. After shearing for a range of accumulated strains, $\gamma_{R}$, the direction of disk rotation is reversed and the sample sheared in the opposite direction until the original azimuthal position of the disk is achieved. We find that for large enough $\mathrm{Er}_{R}$ and for $\gamma_{R}$, below a critical value, the creation and propagation of twist walls is reversible when the flow direction is changed.

Shown in Fig. 6 is a sequence of video images recording during a flow reversal experiment conducted with $\mathrm{Er}_{R}=17500$ and $\gamma_{R}=150$, conditions yielding remarkable reversibility. Each image contains a viewing area of $3.8 \mathrm{~mm} \times 10 \mathrm{~mm}$, with the sample edge appearing at the left edge of each image and the flow direction being first downward (left column) and then upward (right column). The time sequence begins with the upper left image (blackness indicating homeotropic sample orientation) and proceeds downward in the left column where strains for each image are (a) 0 , (b) 33, (c) 66 , (d) 100 , and (e) 133 . The shear is continued until the accumulated edge strain reaches 150 , although no image for this strain is shown. The reversal of the 
Fig. 6 Full-slit observations during the flow-reversal experiment for the case $\mathrm{Er}_{R}=17500$ and a final strain of $\gamma_{R}=150$. Photographs are of the sample viewed between crossed-polarizers, with the polarizer oriented in the flow direction, which is vertical. The viewing area extends from the cell edge (left) to a radial position which is approximately $1 / 4$ of the disk radius. The left column shows photographs taken during counter-clockwise (forward) rotation of the bottom disk for edge strain, $\gamma_{R}$ increasing top to bottom: 0, 33, 66, 100, 133. The shear continued to 150 , at which point the direction reversed. The right column shows photographs taken during clockwise (reverse) rotation. The photo at the bottom right follows in succession after that on the bottom left and the progression continues up the right side as the accumulated strain is reduced from bottom to top: 133,100 , 66,33 , and 0
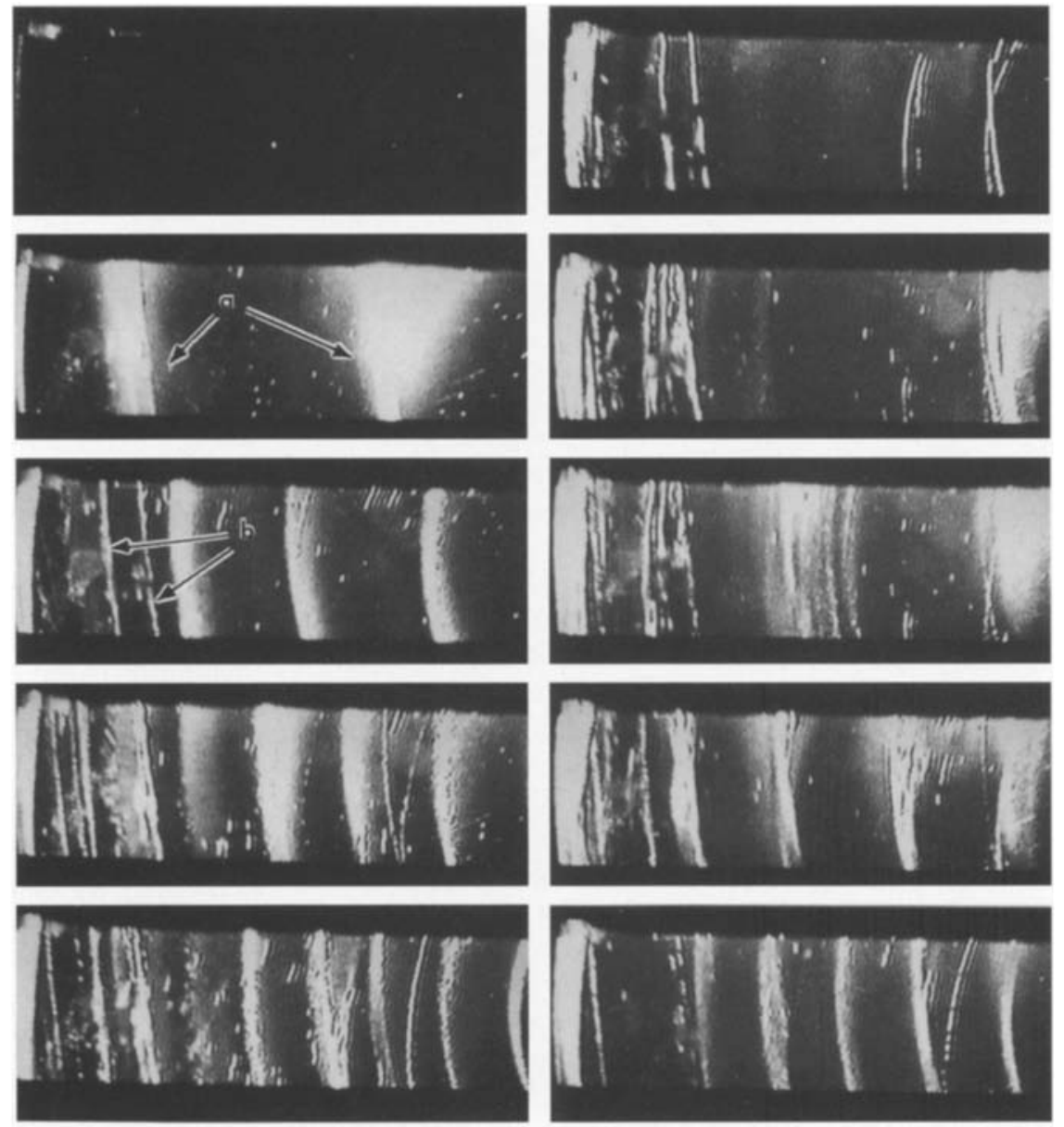

$4 \mathrm{~mm}$ shear flow is shown in the right column with time increasing upward in the column. Strains for these images are (f) 133 , (g) 100 , (h) 66, (i) 33, and (j) 0 . The bright rings indicated by "a" arrows in Fig. 6 are twist walls, while the sharper, ragged bright lines are disclinations, indicated by " $b$ " arrows. The distinction was made by viewing the sample without the analyzer in place to reveal the disclination features by themselves. By the time the reversed flow has returned the disk to the original position, all of the twist walls formed by the "forward" flow have retreated to the sample edge and disappeared, with only a few "wake" disclinations remaining.

In sharp contrast, Fig. 7 shows an analogous sequence of images taken for shear flow reversal with $\mathrm{Er}_{R}=10000$ and $\gamma_{R}=500$, conditions yielding irreversibility in the twist wall creation and propagation. In fact, following the flow reversal, the density of defects increases with strain in the reverse direction (decrease of accumulated strain). Also, no outward propagation of twist walls is observed following flow reversal. The lack of reversibility for the second case is associated with the large value of $\gamma_{R}$, with $\gamma_{R}>\gamma_{R}^{\max }$. While $\gamma_{R}^{\max }$ is smaller for smaller $\mathrm{Er}_{R}$, we find that for both $\operatorname{Er}_{R}=17500$ and $\operatorname{Er}_{R}=10000, \gamma_{R}^{\max }$ is less than 500; i.e., for $\mathrm{Er}_{R}=17500$, irreversibility is also obtained for $\gamma_{R}=500$.

Time-dependent shear-stress measurements were made for $8 \mathrm{CB}$ in the RFS 8500 rheometer under conditions similar to those used in the rheological microscope, namely with parallel plates of diameter $50 \mathrm{~mm}$, a sample thickness of $200 \mu \mathrm{m}$, and $T=37.0^{\circ} \mathrm{C}$. Due to experimental limitations, the edge shear rate was restricted to $12.5 \mathrm{~s}^{-1}$. This yields a value of $\mathrm{Er}_{R}=3070$, which is lower than the values achieved in the optical experiments. Two different experiments were performed under these conditions, one with $\gamma_{R}=150$ and the other with $\gamma_{R}=450$, as in the optical experiments which tested for reversibility. Shown in Fig. 8 is the result for $\gamma_{R}=150$, where the shearing direction was reversed multiple times. During the shear start-up portion $(0<\gamma<150)$ dramatic oscillations in the apparent viscosity are ob- 
Fig. 7 Full-slit observations during the flow-reversal experiment for the case $\mathrm{Er}_{R}=10000$ and $\mathrm{fi}-$ nal strain $\gamma_{R}=500$. Viewing conditions are the same as for Fig. 6 with accumulated strain values of $0,50,220,330,440$ reading from top to bottom for both the forward (left) and reverse (right) cases. The left column shows photographs taken during counter-clockwise (forward) rotation of the bottom disk for edge strain, $\gamma_{R}$, increasing top to bottom: $0,50,220,330,440$. The shear continues to 500 strain units, at which point the direction reversed. The right column shows photographs taken during clockwise (reverse) rotation for strain decreasing from bottom to top: $440,330,220,50$, and 0
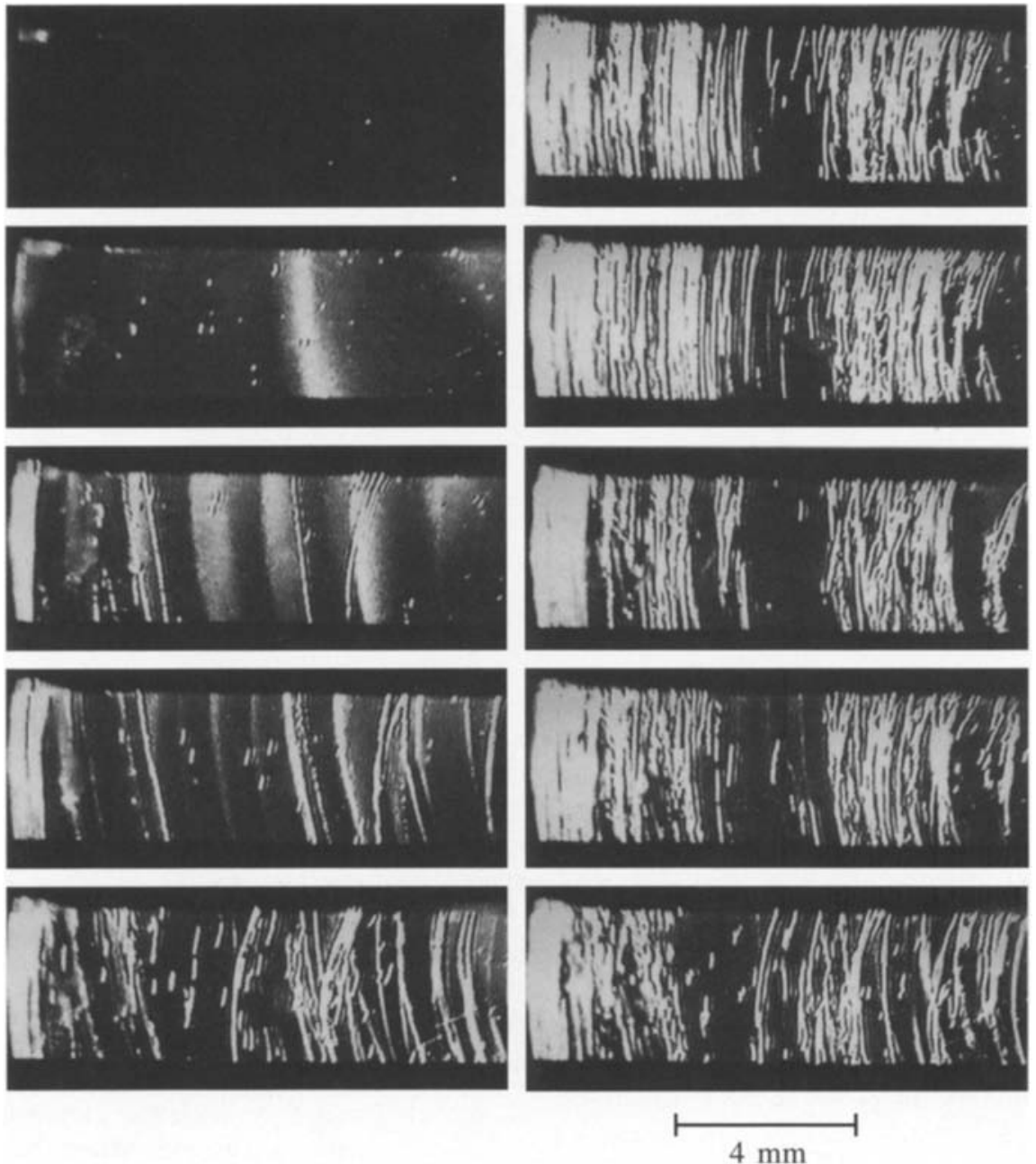

served, similar to those in the cone-plate case of the same material ( $\mathrm{Gu}$ and Jamieson, 1994). The oscillations dampen with increasing accumulated strain and completely disappear at a strain of approximately 100 strain units, yielding an apparent steady-state viscosity of 230 centipoise. On reversal of the torsional shearing direction, small oscillations in the apparent viscosity return in a manner symmetric about the time of reversal. The smaller amplitude of the oscillations that return after reversal, as compared to the start-up oscillations, is probably due to the formation of disclinations, as shown in Fig. 6. Additionally, the $\mathrm{Er}_{R}$ value of 3700 is probably not high enough to eliminate elastic effects, or Er dependencies, which are not accounted for in the Leslie-Ericksen calculations predicting the continued oscillations in rectilinear flow.

Shown in Fig. 9 is a plot of shear stress versus time for the case where $\gamma_{R}=450$, with all other conditions being the same as in Fig. 8. For this larger accumulated strain prior to reversal, no oscillations in the apparent viscosity return on reversal of the shearing direction. This result is consistent with the observation in the rheological microscope under similar conditions (shown in Fig. 7) that at large strains following shear start-up there is a large increase in defect density throughout the shear cell and the density continues to grow on reversal of the flow direction. Also it was observed that for $\gamma_{R}=500$ no outward motion of twist walls on flow reversal was observed, in contrast to the case where $\gamma_{R}=150$.

We see from Figs. 8 and 9 that shear stress oscillations present during shearing prior to reversal dampen rapidly, with the oscillations being completely absent after an edge strain of $\gamma_{R}=75$ has accumulated. This indicates that damping in torsional shearing flow occurs somewhat more quickly than in cone-plate flow, where oscillations continue up to $\gamma \approx 100$ (Gu, Jamieson, and Wang, 1993). The most immediate reason for this contrasting behavior is the gradient of strain present in the plate-plate torsional shearing geometry. In particular, it seems possible that the damping of shear stress oscillations arises from interference of shear stress contributions derived from director orientation angles (profiles) 
Fig. 8 Apparent viscosity versus strain for start-up and reversal of steady shearing in a parallel plates rotational rheometer. The edge shear rate is $12.5 \mathrm{~s}^{-1}$, the sample thickness is $200 \mu \mathrm{m}$, and the plate diameter is $50 \mathrm{~mm}$, yielding $\mathrm{Er}_{R}=3070$. The accumulated edge strain prior to reversal is 150 strain units

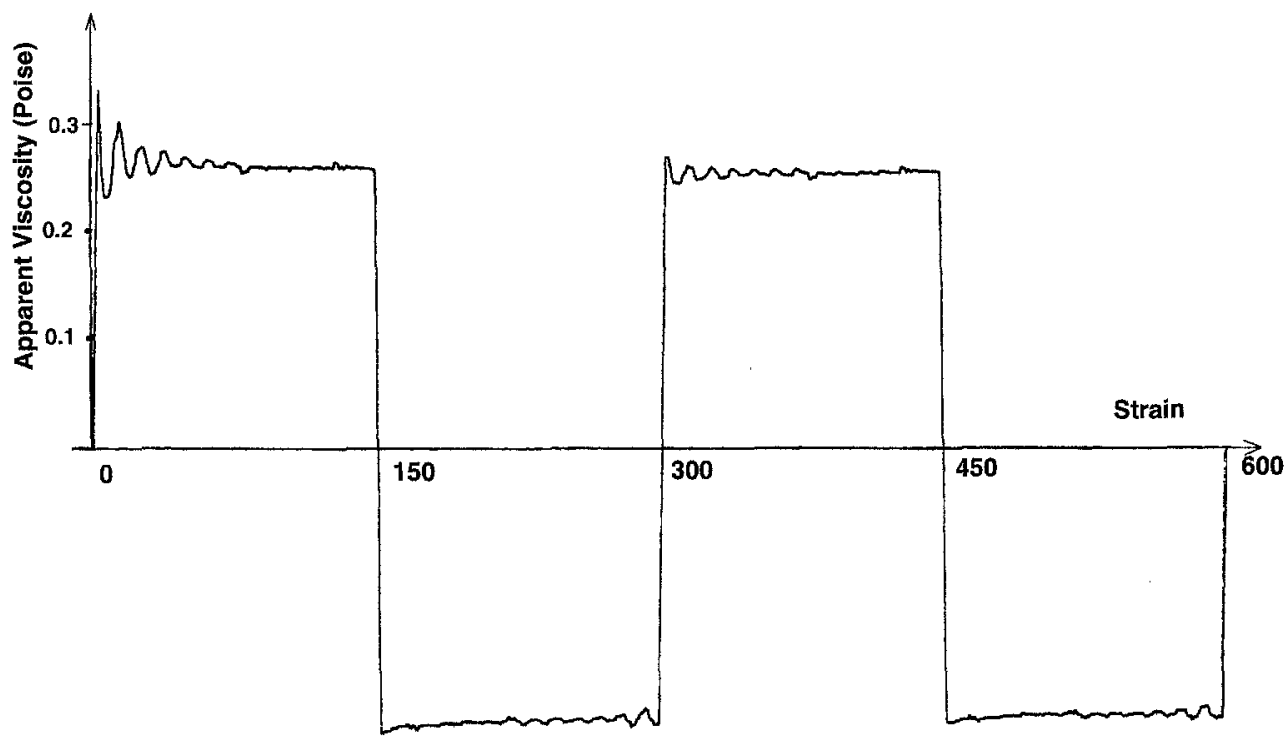

that are non-uniform in the cell. The stress at each position in the cell is oscillatory, but with a phase that depends on strain and therefore on position in the cell. The stress averaged over the sample therefore has an oscillatory character that dampens out as the stress becomes increasingly nonuniform at high strains.

To test this explanation, we perform numerical calculations using the Ericksen's transversely isotropic fluid (TIF) model, which is equivalent to the Leslie-Ericksen model in the limit of large Ericksen number. In the TIF model, the shear stress is given as,

$\tau=\dot{\gamma}\left(\left[a_{1}+\frac{\left(a_{2}+a_{3}\right)^{2}}{a_{3}-a_{2}}\right] \sin ^{2} \theta \cdot \cos ^{2} \theta+\eta_{b}-\frac{a_{3}^{2}}{a_{3}-a_{2}}\right)$

and the director orientation angle, $\theta$, assumed uniform across the sample thickness (negligible elastic effects), is given by

$$
\frac{\partial \theta}{\partial t}=\dot{\gamma}\left(\frac{a_{3} \sin ^{2} \theta-a_{2} \cos ^{2} \theta}{a_{3}-a_{2}}\right) .
$$

Here, the director orientation angle, $\theta$, is taken as zero for orientation normal to the shearing surfaces. The coefficients $a_{1}, a_{2}, a_{3}$, and $\eta_{b}$ are all viscosities appearing in the Leslie-Ericksen equations. Note that Eqs. (4) and (5) neglect any departure of the director from the shearing plane. To compute the apparent viscosity, Eqs. (4) and (5) are discretized in time and space and the torque at each point in time is calculated by integrating shear stress radially,

$$
T=2 \pi \int_{0}^{R} \tau(r) r^{2} d r
$$

The apparent viscosity is then calculated from:

$$
\eta_{\text {app }}(t)=\frac{3}{2 \pi R^{3}} T(t)
$$

The viscosity coefficients appearing in Eqs. (4) and (5) were taken from previous work on $8 \mathrm{CB}$ at $37^{\circ} \mathrm{C}$. We found, however, that, for the best fit to the experimental data, minor adjustments to these previously determined parameters were required.

Fig. 10 shows the results of the calculation along with the experimentally measured apparent viscosity. It is clear that the measured shear stress oscillations and their decay are in nearly quantitative agreement with the predictions, except at strains greater than 50 or so. This provides strong support for the idea that the damping of the shear stress oscillations can be largely explained by a simple in-plane TIF calculation which accounts for the gradient of strain present in the parallel disk shearing geometry. Shown in Table 2 are the values of the viscosity coefficients used in the calculation; while slightly different from those reported previously, they are within the limits of error expected for such measurements.

Since the measured apparent viscosity versus strain can be nearly quantitatively predicted by a calculation that neglects out-of-plane director orientations, a question then arises regarding the observed twist walls and to what extent they affect the rheological observations. Plotted in Fig. 11 is the calculated (in-plane) director orientation angle versus strain, or radial position in the torsional shear cell, for shearing time yielding an accumulated strain at the edge of 37.5. As is expected from Eq. (5) for $a_{3} \ll a_{2}$, the director rotates more rapidly with increasing radius at orientations near $0, \pi, 2 \pi$, etc. This leads to a continuous ring-like pattern within the 
Fig. 9 Apparent viscosity versus strain for start-up and reversal of steady shearing for conditions identical to those in Fig. 8, except using a strain prior to reversal of 450 strain units

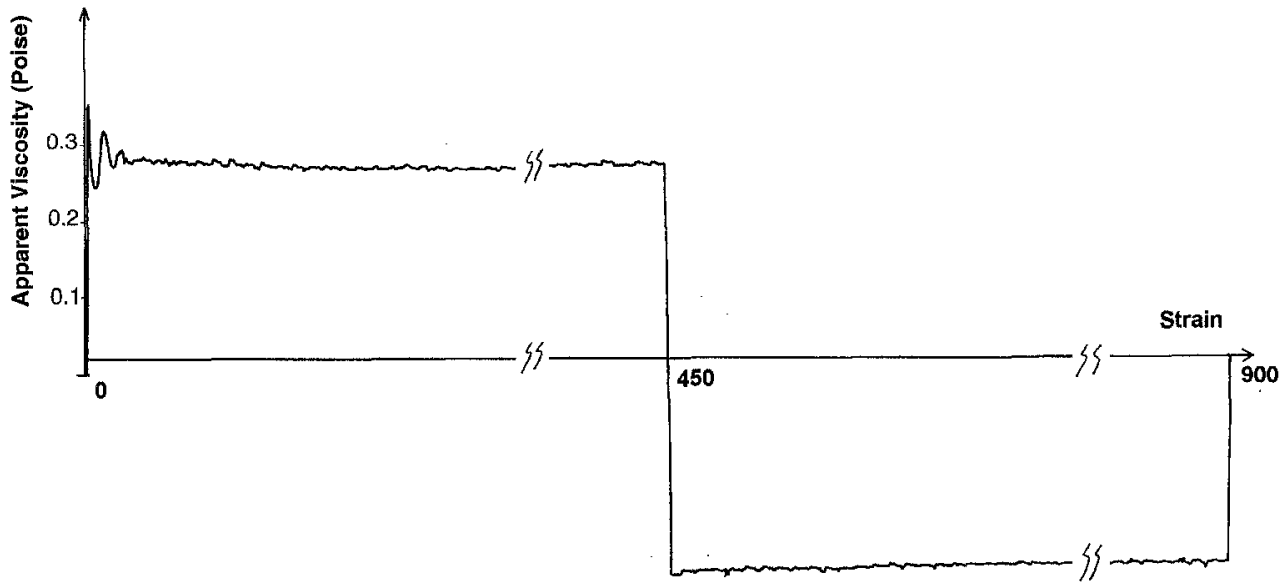

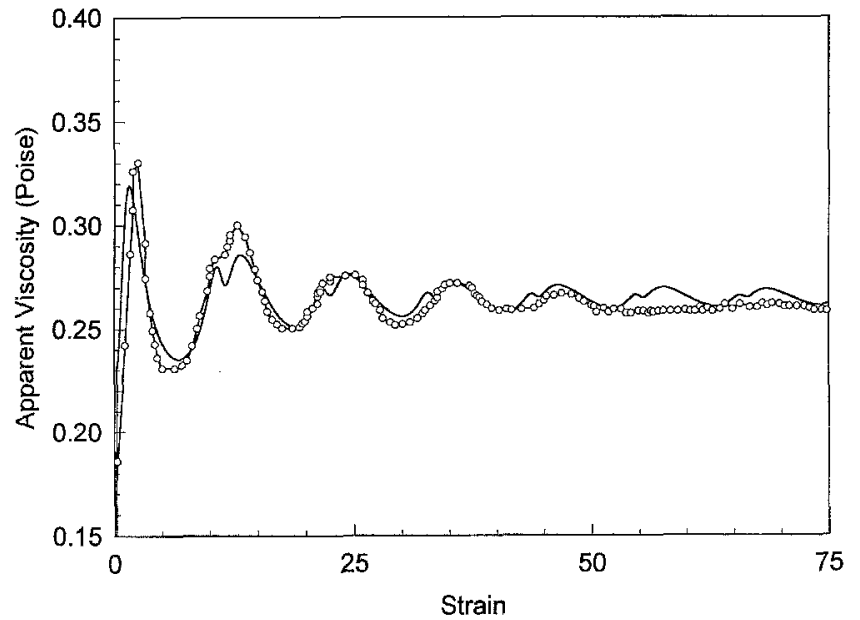

Fig. 10 Apparent viscosity versus strain measured in a parallel plates rotational rheometer (O, same data as Fig. 9) and calculated using the transversely-isotropic-fluid (TIF) model accounting for the strain gradient present in the cell. Parameters used in the calculation are summarized in Table 2

torsional shearing cell in which at any point in time most of the sample is oriented near the flow direction. Also shown in Fig. 12 are vertical lines indicating the critical strain values (Table 1, Fig. 5) where the first and second twist walls were observed. In agreement with the conoscopic observations of the director rotation near the first twist wall, an instability of the director orientation appears to occur near $\theta=3 \pi / 2,5 \pi / 2, \ldots$. Of course, these simple calculations are not able to predict the interesting out-of-plane motion of the director that occurs near these values of $\theta$.

Because the simple calculations predict the stress oscillations and their damping with increased strain rather well (at least for $\gamma<50$ ), the director field throughout most of the sample must be fairly close to that predicted by the in-plane calculations. Thus, apparently,
Table 2 Leslie-Ericksen parameters for shear stress calculation (Fig. 10)

\begin{tabular}{lllll}
\hline Parameter & $a_{1}$ & $a_{2}$ & $a_{3}$ & $\eta_{b}$ \\
\hline Value (poise) & 0.06 & -0.58 & 0.058 & 0.31 \\
\hline
\end{tabular}

only within the twist walls is there a large deviation from the in-plane prediction shown in Fig. 11. Hence, we infer that, at orientation angles of $3 \pi / 2,5 \pi / 2, \ldots$, the director is susceptible to the analog of a mechanical buckling instability that takes the director away from the shearing plane locally, but elsewhere leaves the director field alone. The twist walls that form from the "buckling instabilities" are too narrow to affect the apparent viscosity very much, and so in a gross sense merely act as convenient markers, showing where the director has approached the in-plane angles $3 \pi / 2,5 \pi / 2$, etc. It should in principle be possible to verify this deduction using more rigorous axisymmetric solutions of the full Leslie-Ericksen equations with prescribed homeotropic boundary conditions, and allowing for out-ofplane director orientations.

Note, in Fig. 10, that, for $\gamma>50$, the measured stress oscillations die away essentially completely, even though the simple theory predicts that measurable oscillations should still be present. It is possible that, at these higher strains, deviations from the simple theory are becoming important even outside the twist walls, due to the finite (albeit large) Ericksen number. Indeed, according to Fig. 5, at a strain of $50(t \cong 0.7 \mathrm{~s})$, there is substantial deviation between the measured twist-wall locations and those predicted by the simple theory (dashed lines).

Finally, we note that Han and Rey (1995) have proposed a mechanism for decay of stress oscillations in shearing flow of a tumbling nematic different from that described here. Han and Rey's mechanism was obtained from numerical calculations using the full Leslie-Erick- 


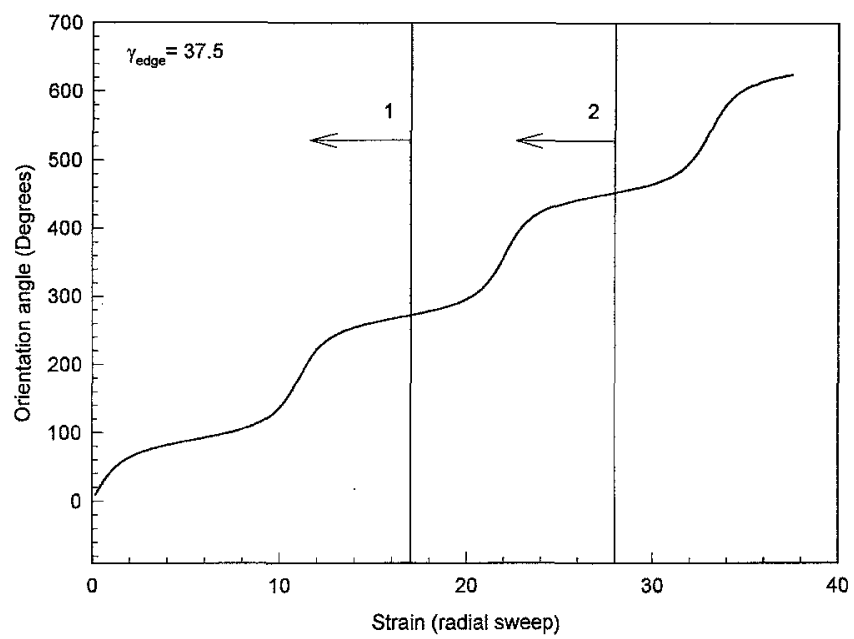

Fig. 11 Orientation angle versus strain $(\gamma)$ predicted by the transversely-isotropic-fluid model, with parameters of Table 2 . In a torsional shearing flow, the plot represents the radial distribution of the orientation angle, with $\gamma=37.5$, corresponding to the sample's edge and $\gamma=0$ to the axis of rotation. The vertical lines mark the experimentally observed locations of the first and second twist walls

sen theory in a plane-Couette geometry. In this geometry, both the strain and the Ericksen number are uniform, and the decay of stress oscillations is produced by an instability that takes the director out of the shearing plane uniformly within the plane of the sample. Thus, in the Han/Rey mechanism, if one were to view the sample along an axis parallel to the velocity gradient, one would see no twist walls, only a uniformly increasing reorientation of the director out of the shearing plane. We see no evidence for this mechanism in the torsional plate-plate flow studied here. However, in a plane Couette flow, there would be no gradient in either strain or Ericksen number, and so the Han/Rey mechanism would apparently be the only one that could produce stress decay. The Han/Rey mechanism may also be relevant for cone-plate flow and, indeed, some of the features of the stress decay observed in this geometry can be explained by the simulations of Han and Rey. However, the stress decay predicted by Han and Rey is much slower than that observed experimentally in coneplate flow, and it seems questionable whether the Han/ Rey mechanism could produce the reverse damping seen experimentally when the direction of the shearing flow is reversed. Thus, a mechanism involving twist walls produced by a gradient in Ericksen number might instead be the dominant mechanism in cone-plate shearing. Direct visualizations in the cone-plate geometry are needed to resolve this issue, however.

We may summarize the above paragraph by noting that there are in principle three sources for oscillatory stress decay in shearing flow of a tumbling nematic: 1) inhomogeneities in strain; 2) inhomogeneities in Ericksen number, and 3) the Han/Rey mechanism. Only mechanism 3) could be relevant in plane Couette flow; mechanisms 2) and 3) could both be relevant in coneplate flow, and all three could potentially be relevant in plate-plate flow. In plate-plate flow, however, we have found evidence only for mechanisms 1) and 2).

\section{Conclusions}

We have shown that the origin of shear stress oscillation damping and reversibility in tumbling nematic liquid crystals in the plate-plate geometry is correlated with the creation and radial propagation of "twist walls." Using conoscopy, the configuration of the director near the twist walls was found to involve localized rotation of the director away from the shear plane followed by rotation within the vorticity-gradient plane to a homeotropic orientation at the defect "core." These observations show that, while the origin of the twist walls may be the inplane tumbling instability predicted by the Leslie-Ericksen theory, the director pattern in the twist wall involves out-of-plane, as well as in-plane, director orientations.

For an Ericksen number of around $10^{4}$, when the shear direction is reversed, if the shear strain $\gamma_{R}$ is less than about 200, the twist walls migrate outward and are expelled from the sample. The damping of the stress oscillations is reversible in this case. If $\gamma_{R} \geq 500$, disclinations form and prevent the reversibility of the twist-wall migration.

Time-resolved image processing of the propagation of the first several twist walls for large Er (17500) reveals that only the first twist wall follows the relationship $r(t) \sim 1 / t$ as expected from the concept of a critical strain describing the propagation. Subsequent twist walls show increasing deviation from this scaling, and we believe this is due to an accumulation of elastic strain caused by the "winding up" of the director at the mid-plane of the sample. Despite the influence of elastic strain, the damping of stress oscillations during the start-up of shearing in the plate-plate geometry can be accurately predicted out to a strain of about 50 by Leslie-Ericksen equations in the infinite-Er limit, by accounting for the radial nonuniformity of the strain, and hence of the director field. Beyond $\gamma=50$, the measured damping of stress oscillations is much greater than that predicted by the infinite-Er theory, evidently because of the accumulation of elastic strain. In a cone-and-plate device, on the other hand, the strain is uniform, so the much more gradual damping of shear stress observed in this geometry is probably due only to the accumulation of elastic strain.

Acknowledgment We are grateful to Annette Shine for suggesting experiments for Fig. 6 and Fig. 7 to RGL, and to Wesley Burghardt for valuable discussions. 


\section{References}

Bloss FD (1961) An introduction to the methods of optical crystallography. Holt, Rinehart and Winston, New York

Burghardt WR, Fuller GG (1990) Transient shear flow of nematic liquid crystals: manifestations of director tumbling. J Rheol 34:959

Carlsson T (1984) Theoretical investigation of the shear flow of nematic liquid crystals with the Leslie viscosity $a_{3}>0$ : Hydrodynamic analogue of first order phase transitions. Mol Cryst Liq Cryst 104:307

Carlsson T, Skarp K (1986) Observation of the tumbling instability in torsional shear flow of a nematic liquid crystal with $a_{3}>0$. Liq Cryst 1:455

Gu D-F, Jamieson AM, Wang S-Q (1993) Rheological characterization of director tumbling induced in a flow-aligning nematic solvent by dissolution of a sidechain liquid crystal polymer. J Rheol $37: 985$
Gu D-F, Jamieson AM (1994) Rheological characterization of director dynamics in a nematic monodomain containing mesogenic polymers of differing architectures. Macromolecules 27:337

Han WH, Rey A (1995) Simulation and validation of temperature effects on the nematorheology of aligning and nonaligning liquid crystals. J Rheol 39:301

Manneville P (1981) The transition to turbulence in nematic liquid crystals. Mol Cryst Liq Cryst 70:223

Mather PT (1994) Shear flow behavior of tumbling and flow-aligning nematic liquid crystals. PhD thesis, U.C. Santa Barbara

Mather PT, Pearson DS, Burghardt WR (1996a) Structural response of nematic liquid crystals to weak transient shear flows. J Rheol 39:627
Mather PT, Pearson DS, Larson RG (1996b) Flow patterns and disclination density measurements in sheared nematic liquid crystals. I. Flow-aligning 5CB. Liq Cryst 20:527

Mather PT, Pearson DS, Larson RG (1996c) Flow patterns and disclination density measurements in sheared nematic liquid crystals. II. Tumbling 8CB. Liq Cryst 20:539

Pikin SA (1974) Couette flow of a nematic liquid. Soviet Phys JETP 38:1246

Rey A (1993) Stationary bifurcations and tricriticallity in a creeping nematic polymer flow. J Non-Newt Fluid Mech 50:1

Skarp K, Carlsson T, Lagerwall ST, Stebler B (1981) Flow properties of nematic $8 \mathrm{CB}$ : an example of diverging and vanishing $a_{3}$. Mol Cryst Liq Cryst 66:199 